\title{
Caso de estudio, PoeTICs: literacidad, arte y tecnología en la formación docente en la Universidad de Sevilla-España
}

\author{
Elena Guichot-Muñoz ${ }^{1}$, Giulia De Sarlo' y Mar Galera-Núñez ${ }^{2}$ \\ (1) Facultad de Ciencias de la Educación, Dpto. de Didáctica de la lengua y de la literatura, Universidad de Sevilla, c/ \\ Pirotecnia s/n, Sevilla, España (correo-e: eguichot1@us.es; gdesarlo@us.es) \\ (2) Facultad de Ciencias de la Educación, Dpto. de Educación Artística, Universidad de Sevilla, c/ Pirotecnia s/n, Sevilla, \\ España (correo-e: mmgalera@us.es)
}

Recibido Feb. 17, 2020; Aceptado Abr. 20, 2020; Versión final Jun. 1, 2020; Publicado Oct. 2020

\begin{abstract}
Resumen
El objeto de estudio de este artículo es examinar el impacto de una práctica letrada que combina la poesía, la multimodalidad y las tecnologías de la información y la comunicación (TIC) como instrumento de alfabetización real en la formación docente de la Facultad de Ciencias de la Educación de la Universidad de Sevilla (España). La metodología consiste en un acercamiento a través de la observación y el análisis de 275 diarios de aprendizaje y de los productos generados por el alumnado. Los datos se analizan bajo la óptica de los Nuevos Estudios de Literacidad, desde una perspectiva artística y colaborativa. Los resultados muestran cómo la inclusión de esta práctica letrada motiva nuevas formas de entender la lectura y la escritura que repercuten en la posición del alumnado en el acto alfabetizador y en la disolución de la división entre alta cultura y cultura vernácula. Destaca además la importancia de las didácticas específicas en las investigaciones sobre alfabetizaciones.
\end{abstract}

\section{A case study, PoeTICs: literacy, art, and technology in teacher training at the University of Seville-Spain}

\begin{abstract}
The aim of this study is to examine the impact of a literacy practice that combines poetry, multimodality, and information and communication technology (ICT) as a tool for real literacy in the teacher training of the Faculty of Educational Sciences at the University of Seville (Spain). The methodology consists of an approach through the observation and analysis of 275 learning journals and the products generated by students. Data analyzes were performed within the framework of the New Literacy Studies, from an artistic and collaborative perspective. The results show how the inclusion of this literate practice motivates new ways of understanding reading and writing. This has a deep impact on the position of students in the literacy act and on the dissolution of the division between high and vernacular culture. This research also highlights the importance of specific didactics in literacy research.
\end{abstract}




\section{INTRODUCCIÓN}

Hablar de poesía en cualquier etapa de la formación educativa significa enfrentarse a uno de los límites más conocidos de la didáctica de la literatura. A pesar de la gran cantidad de poemarios que se imprimen en España cada año (MECD, 2018), la poesía suele ser uno de los géneros menos amados por el gran público (CIS, 2016), y también uno de los que menos se trabajan en la escuela. En buena medida, esto se debe a la falta de una formación docente específica: el profesorado no suele saber utilizar todo el potencial de la poesía como herramienta de desarrollo lingüístico, emocional y vivencial del alumnado. La hipótesis de este estudio nace de la carencia en los programas universitarios de un uso de la palabra poética como un instrumento de alfabetización real. Por un lado, analizando exclusivamente el ámbito de la literatura infantil y juvenil, se percibe una escasa presencia en los programas universitarios de la universidad española de asignaturas que fomenten el conocimiento o la práctica de una alfabetización literaria (Ibarra y Ballester, 2013).

Por otro lado, en el ámbito de la educación superior, y más aún en la formación docente española, también se percibe una carencia de un tipo de alfabetización que vaya más allá de las prácticas convencionales de lectura y escritura, con algunas excepciones reflejadas en el proyecto Cartografías lectoras y otros estudios de lecturas (Fernández y Martos, 2014). Nos referimos en este caso a estrategias de alfabetización que provoquen una "ruptura cognitiva", similar al fenómeno jakobsoniano de desfamiliarización que se da en el aula para favorecer un proceso educativo transformador (Acaso y Mejías, 2017) por medio del uso de herramientas, instrumentos y metodologías que procedan del ámbito artístico.

En base al aspecto social y emocional de las prácticas realizadas, se relaciona esta actividad con los Nuevos Estudios de Literacidad (NEL; New Literacy Studies) que acentúan el aspecto inmaterial de la alfabetización (Burnett et al., 2014). La alfabetización, por tanto, se encuadra como una práctica social (Knobel y Lankshear, 2007), donde convive la creatividad cotidiana: "poetic, literary, and artistic texts are part of a landscape of outof-school literacies" (Pahl, 2014, p. 83). Indudablemente, entra en juego la tecnologización del mundo, y la emergencia de una alfabetización llamada multimodal, que incluye fuentes verbales, visuales, digitales, sonoras, diferentes modos de acceso y diversos espacios desde el que generarla (Mills y Unsworth, 2017).

A pesar de este panorama innovador, en la formación universitaria española se continúa con un acercamiento obsoleto a las TIC (Tecnologías de la Información y la Comunicación) en la educación superior española (Ramírez y Casillas, 2014) pues se sigue usando en ocasiones como "aderezo cosmético a una metodología tradicional” (Gutiérrez et al., 2003). En concreto, la educación artística continúa prácticamente estancada tras la revolución tecnológica (Acaso y Mejías, 2017), y carente de paralelo con la alfabetización informal del alumnado, usuario de nuevas tecnologías que moldean su identidad (Coiro et al., 2014; Schamroth-Abrams y Rowsell, 2011). No obstante, existe un antecedente novedoso de alfabetización multimodal en España concretamente en la Universidad de Málaga-, aunque se centra en la conjunción del elemento audiovisual y performativo: "Alfabetización Audiovisual para la Práctica Performativa" (Código: PIE17-172; 2017-19). Este proyecto coincide en el objetivo primordial de convertir al alumnado universitario en emisor-constructor de su propio conocimiento desde enfoques tan importantes como la coeducación, la multiliteracidad y el artivismo. Desde la misma coordinación nace el proyecto de investigación LITMEC (Literacidad Multimodal y Estudios Culturales. Hacia una Educación Ciudadana en la sociedad postmoderna) que también tiene como finalidad insertar los principios metodológicos y las corrientes teóricas de Gunther Kress en la formación docente de Ciencias Sociales (Triviño, 2018). En otras universidades españolas también se ha vinculado la creación genuina de podcast educativos y la metodología ABP, aunando las tecnologías y el trabajo colaborativo y autónomo para la adquisición de competencias en la formación pedagógica, con un alto grado de satisfacción de alumnado (Ausín et al., 2016).

En esta misma línea, apostando por la multimodalidad, la digitalización y la utilización artística de los distintos lenguajes verbales y no verbales, desde la Facultad de Ciencias de la Educación de la Universidad de Sevilla se ha elaborado y llevado a cabo una intervención docente de escucha y creación poética conectada con las nuevas prácticas digitales, en consonancia con proyectos como los de Curwood y Cowell (2011). La intervención, titulada "PoeTICs", surge a su vez dentro del Proyecto de Innovación Docente: La Investigación Basada en las Artes: una nueva metodología para la aprehensión del conocimiento en la formación inicial del profesorado, y del proyecto de investigación El impacto de la revolución digital, y de la cultura popular y de masas en los procesos de alfabetización (Ref.: CAS18/00469), que se inserta asimismo en el Proyecto I+D "La alfabetización como práctica social en educación infantil y primaria (5-7 años): Investigación y diseño de intervención en niños con riesgo de exclusión social en contextos urbanos", financiado por el Ministerio de Economía, Industria y Competitividad de España (Plan de Excelencia 2018-2020, código EDU2017-83967P). La filosofía que mueve el proyecto es la búsqueda de una nueva experiencia de enseñanza-aprendizaje en la que los lenguajes artísticos, independientemente de la disciplina que se esté impartiendo, puedan vehicular no solo una experiencia discente sino un aprendizaje profundo que permita al alumnado romper las barreras a menudo impuestas por el contexto académico, y utilizar el espacio universitario y sus dinámicas como un motor de descubrimiento de sus propias potencialidades creativas y comunicativas. 
Se parte de una idea nueva de alfabetización que va mucho más allá del mero aprendizaje lectoescritor y del desarrollo de una cierta familiaridad con los géneros literarios tradicionales, moviendo la atención hacia el evento alfabetizador y hacia las prácticas letradas. En relación al ámbito estrictamente artístico, se supera asimismo la teoría de la metáfora conceptual de Lakoff y Johnson, que apunta al lenguaje verbal como sistema de significación, "ignorando que el pensamiento metafórico puede valerse de otros modos semióticos como '(1) the pictorial or visual mode; (2) the aural or sonic mode; (3) the olfactory mode; (4) the gustatory mode; and (5) the táctil mode' (Forceville y Urios-Aparisi, 2009, p. 22).

En este sentido, Burnett y Merchant (2018) proponen que el verdadero objeto de estudio debe ser el acontecimiento alfabetizador (Literacy-as-Event): es decir, el poema por sí solo no es fuente de alfabetización, este solo cobra vida en el encuentro con el lector en un determinado espacio, y dicho evento tendrá un sentido muy específico e irrepetible. La investigación se centra en el tipo de relación que se da entre los objetos, los espacios y las personas en el evento alfabetizador. La atención entonces va: on what happens as people and things come into relation rather than as separate pre-existing entities (...) it is never an isolated activity, but is always in relation with other people and things" (Burnett y Merchant, 2018, p. 2).

El encuentro entre el sujeto y la palabra escrita y leída es entonces el verdadero momento alfabetizador, así como las dinámicas que se crean, únicas e irrepetibles, en un entorno que no excluye sino más bien favorece la implicación emocional de todos los participantes. Como anota Massumi, "It is important not to confuse the event with a state of things, with bodies and materials that come together to produce results (...) the event is the effect of their mingling and colliding" $(2015,66)$. No es el resultado en sí donde está el foco: la intervención didáctica puede ser susceptible de replicar pero el verdadero objeto de análisis será el encuentro entre el poema y el alumno, y cómo el evento alfabetizador genera un "turn to affect" (Leander y Ehret, 2019), es decir: ¿cómo interactúan afectivamente las personas con la materialidad e inmaterialidad de los textos? En esta línea, en nuestro marco metodológico se incluyen nuevos acercamientos al evento alfabetizador como el de Leander y Ehret (2019). Estos autores muestran innovadoras vías de análisis que se centran en el afecto en el proceso de enseñanza-aprendizaje del fenómeno literario, haciendo hincapié en la singularidad del evento como una mezcla vibrante e irrepetible de cuerpo, afecto, materia, sociedad y fuerzas semióticas (Massumi, 2015).

Además, el tipo de creaciones que realiza el alumnado se corresponden con lo que Knobel y Lankshear (2007) definen como literacidades emergentes que combinan el arte y la alfabetización dentro de blogs y podcasts. Estos productos requieren un cambio de mentalidad en la forma de entender alfabetización puesto que suponen una nueva interpretación, lectura de lo tradicional: "traditional literacy (reading and writing) in creating print-based texts, and unpredictable literacy, which promotes reading and writing through multimedia (paper, the arts, blogs, software programs [iMovie, Photoshop, Movie Maker, Garage Band, and so on]) to create morphed and altered texts" (Albers y Harste, 2007, p. 16). Siguiendo las teorías de estos autores y de otros como Rowsell y Pahl (2015) se combinan en este artículo los conceptos de arte, multimodalidad y los NEL para mostrar una implementación didáctica en el aula que favorece un acercamiento contemporáneo al currículum y una perspectiva inclusiva de la educación.

En segundo lugar, analizamos la práctica letrada como una práctica cultural, entendida por Rockwell en relación a cuatro ejes: "La materialidad, las maneras de leer, las creencias y las prácticas orales que envuelven al texto" (2001, p. 19). Por tanto, esta intervención se analizará en base a los NEL, tratando de resolver si nos encontramos ante un evento alfabetizador (Burnett y Merchant, 2018), y ante una práctica letrada contemporánea. Para ello, resolveremos las siguientes afirmaciones que conforman las bases de este reciente acercamiento teórico de Burnett y Merchant (2018, p. 1): (1) El evento se genera a medida que las personas y las cosas se relacionan (2) lo que sucede siempre excede lo que se puede concebir y percibir (3) implícitamente en el evento están las múltiples potencialidades, incluyendo las múltiples posibilidades de lo que podría materializarse así como lo que no.

\section{METODOLOGÍA}

En base al marco teórico proporcionado, se lleva a cabo un acercamiento etnográfico que implica una documentación y observación de la intervención didáctica en el aula, desde un punto de vista ecológico y participativo (Pahl y Allan, 2011). Por esta razón, analizamos un conjunto de prácticas letradas (Bertely, 2000) que derivan de la intervención didáctica.

\section{Contexto y participantes}

El contexto del estudio es la Facultad de Ciencias de la Educación de la Universidad de Sevilla (España), en concreto las prácticas de 5 grupos de estudiantes: dos grupos de $2^{\circ}$ curso del grado de Educación Primaria para el año académico 2017/2018, un grupo de $4^{\circ}$ año del grado de Pedagogía para el año académico 2018/2019, y dos grupos para el año académico 2019/2020 del grado de Educación Primaria. Participaron un total de 275 alumnos/as. 


\section{Descripción del procedimiento}

El trabajo de campo contempla dos fases: 1) Descripción de la intervención didáctica y 2) Evaluación de la intervención por parte del alumnado.

\section{Fase 1: Descripción de la intervención didáctica}

"PoeTICs" se plasmó en la realización de un taller que consta de dos partes. La primera consiste en que en un día normal de curso, sin ninguna preparación previa, los/as alumnos/as llegan a clase encontrando las paredes del aula decoradas con poemas, impresos en blanco y negro en simples folios A4, sin ningún tipo de indicación relativa al autor/a, el título de la obra o la fecha de publicación. Se trata de un corpus de 11 poemas de diferentes autores/as. Las profesoras crean esta antología siguiendo criterios de afinidad con los intereses del alumnado. Teniendo en cuenta el margen de edad y la especialidad universitaria se escogen poemas de temática existencial, amorosa y concerniente al tema de la educación. Además, se eligen autores/as de distinto país, género y época para que contemplen cierta heterogeneidad de estilos. No obstante, en una experimentación sucesiva podría perfectamente ser modificado para adaptarse al grupo. Con una base sonora que invite a la meditación y el recogimiento, el alumnado es invitado a levantarse de sus asientos y moverse por el aula, leyendo en silencio los poemas. Una vez terminada la lectura, se les invita a elegir el poema con el que más se hayan sentido en consonancia; el alumno o alumna elige luego, entre todos los compañeros, a una pareja a la que conozca lo menos posible: le cierra los ojos y la lleva de las manos hasta el poema elegido a modo de lazarillo, leyéndoselo en voz baja. Luego la experiencia se repite al revés. La actividad se cierra con una puesta en común.

La segunda actividad del taller es grupal, y se realiza a lo largo de tres sesiones sucesivas. Se pide a cada grupo que realice un nuevo poema a partir de las obras leídas en la primera actividad, fragmentando versos, estrofas, buscando nuevos esquemas métricos, y añadiendo nuevos textos originales o tomados de su propio bagaje alfabetizador, siguiendo una inspiración colectiva. Una vez creado, el nuevo poema es leído por el grupo de forma coral (no necesariamente al unísono pero sí haciendo que todas las voces estén de alguna manera presentes), pudiendo ser acompañado por sonidos ambientales, bases musicales o imágenes. La lectura se graba y se edita con programas de software libre (Audacity, Movie maker, filmora), y se sube a la plataforma Soundcloud generando una nueva galería poética, esta vez sonora: https://soundcloud.com/elenaguichot

\section{Fase 2: Evaluación de la intervención por parte del alumnado}

La evaluación se llevó a cabo a través de la revisión de un diario de prácticas o un blog virtual (diario de aprendizaje) con unos criterios de evaluación expuestos en una rúbrica y consensuados con el alumnado. Estos criterios estuvieron basados en los conceptos de multimodalidad, creatividad de estilo de escritura, complejidad en la reflexión sobre el desarrollo de la práctica letrada como práctica cultural y reflexión sobre la escritura. El diario podía tener distintos soportes: cuaderno de papel, blog, cuaderno digital, e-portfolio, etc. Cada día se establecían distintas consignas pero en el diario en general se debía recopilar (al menos) la siguiente información propuesta por Vergara (2017): ¿Qué es lo más importante que has aprendido en esta fecha? Haz una lista de palabras clave del tema y explica por qué lo consideras un aprendizaje importante? ¿Qué preguntas se te plantean en torno a este tema? ¿Qué consecuencias tiene lo que he aprendido en mi vida? ¿Tiene relación con mi vida cotidiana? ¿Cómo cambia mi forma de entender la realidad? ¿Qué te gustaría saber o hacer en relación a lo que has aprendido hoy? ¿Qué tendrías que hacer para conseguirlo? (p. 175)

\section{Técnicas para la recopilación y análisis de los datos}

Consecuente con el modelo etnográfico, las estrategias de recopilación de datos se hicieron a través de la observación y grabación de los eventos, y la lectura y análisis del diario de aprendizaje o blog y de los productos generados por el alumnado. La observación y grabación de las sesiones, y el análisis de los diarios y productos nos proporcionan nuevas claves de lecturas de esta alfabetización del afecto, del aquí y del ahora, en consonancia con lo propuesto por Leander y Ehret (2019): "queremos contar en voz alta el secreto que los maestros parecen saber, que la mayoría de lo que sucede, en nuestros mejores días, no puede ser explicado en marcos racionales. Estamos conmovidos, y nuestros estudiantes están conmovidos, y no podemos explicar cómo o por qué" (p. 2). Respecto al modo de análisis, se revisan las declaraciones del alumnado en base a los enunciados del objeto de estudio, y se lleva a cabo una serie de relaciones conceptuales sirviéndonos de la teoría de las NEL, con el fin de dar luz sobre este nuevo enfoque de la literacidad y su relación con la formación docente. 


\section{RESULTADOS Y DISCUSIÓN}

Como ya avanzamos, nuestro objeto de estudio es dilucidar y examinar si estamos ante un evento alfabetizador y una práctica letrada contemporánea, y de qué modo. En este sentido, se analizan las reflexiones del alumnado según las premisas de Burnett y Merchant (2018) que nos permitimos traducir al castellano.

\section{El evento se genera en la medida en que las personas y las cosas se relacionan}

El alumnado, casi en su totalidad, contempla la riqueza de esta vivencia subrayando el encuentro con los materiales artísticos y la convivencia entre los compañeros: la verdad es que me encantó leerle mi poesía favorita a mi compañera porque le puse mucho sentimiento, pero sobre todo me encantó cuando me la estaba susurrando ella a mí, pues pienso que al tener los ojos cerrados fue como si toda mi atención se centrase en su voz. Me transmitió muchísimo, fue una experiencia que nunca antes había sentido (sujeto 2 ).

Muchos de los participantes aluden a la sensación de presencia y de fluidez, característica que los NEL ponen en primer plano, refiriéndose a la necesidad de que se dé la cualidad de vivacidad en las prácticas de alfabetización, con el fin de abarcar lecturas de lo social y cultural en el evento alfabetizador (Burnett y Merchant, 2018). De hecho, el científico Csikszentimihalyi acuñó el término de "fluidez" para describir aquellos momentos en los que nuestra atención se centra en una tarea determinada, relegando el resto de los pensamientos a un lado, con el consiguiente aumento de conciencia sobre el acto realizado: "La experiencia del flujo se caracteriza por la pérdida de la conciencia de sí mismo, un sentido distorsionado del paso del tiempo y la fusión de la acción y la conciencia" (Csikszentmihalyi y Nakamura, 2018, p. 102). En este sentido, tras la experiencia de la intervención, una alumna reflexiona sobre su vivencia de la poesía en el aula: los niños no acostumbran a jugar con la poesía y no hablan ni cantan poesía. Hace poco leí que "el aprendizaje hace ruido" y estoy totalmente de acuerdo, el aprendizaje es acción, movimiento, cambio y "jaleo", en el buen sentido de la palabra. Por tanto, la poesía también debería ser aprendizaje académico y aprendizaje de vida (sujeto 121).

\section{Lo que sucede siempre excede aquello que se puede concebir y percibir}

La mayoría del alumnado muestra una superación de las expectativas sobre su encuentro con el poema, debido a la multimodalidad de la propuesta. Esta experiencia me ha resultado más satisfactoria de lo pensado, y al leer en silencio cada uno de los poemas, he podido conectar con mi subconsciente dándole a cada uno el sentido que más me apetecía. En definitiva, supongo que de eso se trata, de interpretar el significado de los poemas de forma individual y muy íntima (sujeto 54). Aunque recitarlo sea reconfortante, aún más lo es escuchar un poema con los ojos cerrados (sujeto 87). Ha sido precioso, cuando me ha tocado leerle el poema me ha gustado mucho más, tener que guiarla hasta él y que confiara en mí ha sido una experiencia muy bonita porque era alguien a quien no conocía y mediante ese gesto de "protegerla" le he dicho que confíe en mí, que no pasará nada (sujeto 135).

Se da una transformación completa del concepto de alfabetización (Bearne y Wolstencroft, 2007) pues se asocia la literacidad con una naturaleza más participativa, colaborativa, democrática, tanto en su recepción como en su emisión. El alumnado percibe que las prácticas no están sujetas a reglas específicas, por tanto el evento de la práctica poética es "more fluid and less abiding than those we typically associate with established literacies" (Knobel y Lankshear, 2007, p. 9). El alumnado llega a percibir la capacidad de que el evento alfabetizador sea una práctica social y cultural: Esta actividad, me ha hecho pensar y darme cuenta de que podemos expresar y transmitir diversidad de cosas a las personas a través de estos ejercicios y a través de nuestras palabras. Me parece que es una buena forma de perder el miedo de expresar lo que sentimos y que nos sintamos libres de crear frases, versos, cuentos, canciones, discursos con nuestras palabras (sujeto 213).

En el evento están implícitas múltiples potencialidades, incluyendo diversas posibilidades de lo que podría materializarse y de lo que no. Las preguntas de reflexión que encontramos en muchos de los diarios nos sugieren otros elementos de análisis: ¿Por qué me ha gustado más y he sentido una mayor atracción por el poema cuando la otra persona me lo ha leído? En mi opinión, creo que esto se debe a que R. lo ha leído con especial cariño y supongo que me ha transmitido tanto ya que ella lo ha leído expresando todo lo que ha sentido al leer el poema. Otra de las preguntas que se me vienen a la cabeza es ¿puedo llegar a sentir lo mismo que he sentido cuando R. me ha leído el poema si lo leo yo? (sujeto 26)

Las conexiones que los propios alumnos/as nos proponen parten de la experiencia creativa tal y como se planteaba en el proyecto de aprendizaje basado en lenguajes artísticos, pero se alargan hasta una visión más general de la experiencia, que la vincula a los estudios más recientes en el ámbito de los NEL, tanto por la 
resemantización del objeto de transmisión alfabetizadora como por la importancia que se da al propio acontecimiento alfabetizador. En primer lugar, el objeto: el poema ya no es un tótem cultural inamovible, ubicado en un libro impreso, sino que sale del volumen, y del conjunto para el que había sido pensado y con el que había sido tradicionalmente difundido, y encuentra una nueva ubicación, siguiendo el fenómeno denominado "transcontextualidad" que implica un acercamiento colectivo y dentro y fuera de la pantalla del objeto artístico (Davies, 2014). Es más: la elección de obviar cualquier dato bibliográfico en la presentación inicial de los poemas desmonta en un cierto sentido la propia autoridad del texto (Kell, 2015), identificando este como objeto alfabetizador solamente en la medida en que se le ubica en unas coordenadas temporales y espaciales determinadas. La misma dinámica de ruptura, de abandono del estatus estanco del objeto poético, se ha obtenido en la segunda parte de la actividad en la que, siguiendo las sendas surrealistas, los versos han sido desmontados y reconstruidos por los/as alumnos/as, obteniendo en algunos casos resultados muy interesantes desde el punto de vista textual, musical y hasta plástico (Fig. 1).

\begin{tabular}{l}
\hline El lenguado y la cebolla \\
Incansablemente \\
toda la arena, todo el vasto fondo marino \\
hambre \\
y sentir un sabor \\
se amamantaba. \\
Na felicidade \\
dentadura cuando en la \\
Los amorosos son insaciables \\
No te derrumbes tú, satisfecho \\
las tripas los ojos aleante \\
La carne aletea \\
Boca que vuela \\
les llega a veces un olor \\
se los comen los... \\
un pequeño monstruo invisible \\
creen que las devora \\
and look! \\
las breves anchovetas que pasan a mi \\
lado
\end{tabular}

Fig. 1: Poema elaborado por alumnado (Autorizada, realizada en la FCCE de la Universidad de Sevilla).

En otras ocasiones el poema es intercalado por textos de otras procedencias como es el caso de la siguiente alumna: El trabajo que he realizado junto a mi compañera $X$ a lo largo de esta semana ha estado basado en la recopilación de lecturas, películas y fragmentos de texto en base a lecturas de ficción y poemas diversos entre los que destacamos El Señor de Los Anillos (JRR Tolkien), Joker (Todd Phillips), La gente dice (Gloria Fuertes), El Principito (Antoine de Saint-Exupéry) así como pareados de elaboración propia. Como música de base hemos elegido el tema "Evenstar" de la Banda Sonora de la película Las Dos Torres compuesta por Howard Shore, de entre los temas que propusimos que también nos evocaron los sentimientos que íbamos buscando transmitir se encuentran varias composiciones de Hans Zimmer (la canción "In Time" de la banda sonora original de la película Inception) o de Ludovico Einaudi la canción "Experience". Al final por el propio ritmo creemos que la más adecuada ha sido la de Howard Shore, dicho tema es con el que hemos conectado mejor y el que más encaja con el tema propio de nuestro poema, la vida y muerte (sujeto 264).

El poema se convierte en un "artefacto cultural" (Pahl y Rowsell, 2019) que cuenta una historia del propio alumno/a: este usa sus "funds of knowledge" (González et al., 2006) para mostrar su creación original, es decir, vincula el texto impreso canónico con sus herencias, creencias, su cultura, y su alfabetización diaria informal. La multimodalidad del evento alfabetizador ha tenido otra vuelta de tuerca con la última parte de la actividad: transformando sus creaciones en archivos de sonido, los/as alumnos/as han creado nuevos objetos alfabetizadores que se proponen al lector/oyente en un nuevo formato y que a su vez se ponen a disposición para ser interpretados, desmontados y manipulados por el nuevo sujeto de alfabetización. La identidad entra en juego y tanto el maestro/a como el investigador/a pueden contrastar las identidades "escolares" o universitarias con las de fuera del espacio institucional: Conocemos que los poemas son medios de expresión de sentimientos, ideas, valores, pero nunca los hemos tratado como tal. La oportunidad de tomar versos de poemas que te gusten, inspirarte y utilizar nuestra imaginación y creatividad para crear nuestros propios versos es una forma totalmente innovadora y original para trabajar los poemas y hacer que la gente disfrute con ellos (sujeto 203). 
Indudablemente, cuando la metáfora posee carácter multimodal se potencia la interpretación de significados, por lo que la comunicación se dirige a una nueva comprensión de la experiencia y de las acciones humanas (Forceville y Urios-Aparisi, 2009). En recientes investigaciones se habla de trasladar el fenómeno estético de "intermedialidad" a la educación, la creación de "formas híbridas artísticas híbridas" (Masgrau-Juanola y Kunde, 2018, p. 621) para generar en los estudiantes la capacidad de ser tanto consumidores como creadores. Una alumna, por ejemplo, explica lo siguiente: No teníamos muy claro que canción elegir, pero finalmente, nos decidimos por La caravana de los Zincalí de Dorantes, ya que esta canción habla del camino que hicieron los gitanos desde la India a España, perdiendo su antigua vida y dejando todo por tener que irse a otro lugar, algo muy relacionado con nuestro poema, ya que habla del arte de perder (Sujeto 56). La intermedialidad permite que los usuarios puedan entender cuestiones imprescindibles del siglo XXI "como la autoría y la creación colectiva, la diseminación transmedia de los productos culturales (y sus derivadas comerciales) y la edición de contenidos (o la ausencia de edición en la generación y difusión), la obra -abierta" (Masgrau-Juanola y Kunde, 2018, p. 636).

En base a esta teoría, una cuestión muy importante en relación con la digitalización de estos artefactos es que "las redes sociales no son simplemente lugares de intercambio de información, 'también son sitios para la experimentación de identidades' (Lemke 2009a, p. 590)"' (Cárdenas, 2014, p. 178). Si ponemos esto en relación con los procesos de alfabetización nos damos cuenta de que la experimentación de la identidad se da en la escuela en un ámbito muy limitado, mientras que en los procesos informales de alfabetización, gracias a la ampliación de los mundos digitales, nos encontramos con el campo de la heteroglosia, la diversidad, el cambio, tan necesarios para la aceptación y legitimación de multiplicidad de identidades. En este sentido, una alumna vincula esta actividad con un aprendizaje de vida: Hace poco leí que 'el aprendizaje hace ruido' y estoy totalmente de acuerdo, el aprendizaje es acción, movimiento, cambio y 'jaleo', en el buen sentido de la palabra. Por tanto, la poesía también debería ser aprendizaje académico y aprendizaje de vida.

Trasladado al campo de la revolución digital, nos abre un mundo de posibilidades pues se observa cómo la cibercultura empieza a legitimar patrones procedentes de la cultura popular debido a su presencia, accesibilidad y, sobre todo, a su consumo, en especial por parte de los niños (Parry et al., 2017, p. 41). Por otro lado, en relación con nuestra temática concreta, Bourdieu (1987) explicita que otro campo social relevante es precisamente el sistema educativo. Los procesos de alfabetización serían parte del espacio donde los agentes implicados (profesorado, familia, alumnado), pueden ejercer su poder dentro del sistema. El campo (sistema educativo, en este caso) manifiesta su influencia sobre la perspectiva y las acciones de los participantes, por tanto los datos de una valoración y de un juicio. Tal y como afirma una de las alumnas: Creo que el problema de esto es que, desde pequeños, nos han enseñado multitud de poemas en el colegio, pero siempre para que aprendamos a analizarlos, nunca para disfrutarlos y analizarlos de una manera más profunda. Por eso pienso que, a día de hoy, nos puede costar más sentir de verdad un poema y, por eso también, me ha llamado mucho la atención esta forma de trabajarlos (sujeto 135). La perspectiva teórica de una didáctica de la lengua y la literatura dedicada exclusivamente al texto y sus propiedades, y no a la relación con el lector desde la óptica de la emoción, ha influenciado en la concepción sobre la poesía de esta alumna.

Esto también podría observarse, por ejemplo, en la cuestión de los procesos informales de alfabetización y su relación con la cultura popular y de masas, excluidos del sistema educativo y, por tanto, carentes de capital simbólico en el mismo. Si se lleva a cabo la inserción de estos procesos de alfabetización informales, combinados con la guía de la cultura propia del sistema educativo, se puede conseguir una inclusión mayoritaria del alumnado en la formación escolar, y un lugar desde el que trabajar con los procesos de aprendizaje del discente.

\section{CONCLUSIONES}

De acuerdo al trabajo presentado y a los resultados obtenidos, se pueden plantear las siguientes conclusiones principales:

1.- Se puede afirmar que, a través de esta intervención didáctica, los/as alumnos/as han podido acercarse a la palabra poética de una forma personal, emocional y compartida. Se fomenta así un tipo de formación docente que tiene su piedra de toque en la creatividad, con la idea de generar un/a docente capaz de ser flexible e imaginativo en su experiencia particular del aula.

2.- Se apuesta por evitar el aprendizaje mecánico en la formación docente en aras de conseguir espacios dialógicos en la escuela, necesarios para educar a ciudadanos del siglo XXI. En este sentido, se reconocen otras formas de leer y escribir más allá del texto impreso en la formación docente, lo que también supone desafiar la alfabetización verbal e impresa como única base de examen de la capacidad de leer y escribir.

3.- Se asumen otras formas de acceso al conocimiento letrado, con la condición de dar un papel activo del alumnado, que estimule su estatus de ciudadano del mundo (Feigenbaum, 2014). 
4.- Se disuelve la división entre cultura popular y alta cultura, o entre la alfabetización cotidiana o informal y la alfabetización escolar, acabando con la dicotomía entre "prácticas dominantes" y prácticas vernáculas" (Barton y Hamilton, 2012). El maestro/a debe empezar a lidiar con una alfabetización transformadora, adaptada a los tiempos, que coloque al alumno en un lugar de dominio.

5.- Se observa la importancia del uso de la poesía para el aprendizaje de la alfabetización en la formación docente y la necesidad de investigaciones sobre alfabetización desde el ámbito de las propias didácticas específicas, como es el caso de la didáctica de la lengua y de la literatura.

\section{REFERENCIAS}

Acaso, M., y Megías, C., Art Thinking. Cómo el arte puede transformar la educación, Paidós Educación, Madrid (2017).

Albers, P., y Harste, J. C. The arts, new literacies, and multimodality, English Education, 4O(1), 6-20 (2007).

Ausín, V., Abella, V., Delgado, V., \& Hortigüela, D. Aprendizaje basado en proyectos a través de las TIC: Una experiencia de innovación docente desde las aulas universitarias. Formación universitaria, 9(3), 31-38 (2016).

Barton, D., y Hamilton, M., Local literacies: Reading and writing in one community, Routledge (2012).

Bearne, E., y Wolstencroft, H., Visual approaches to teaching writing: Multimodal literacy 5-11, Sage (2007).

Bertely Busquets, M., Conociendo nuestras escuelas: un acercamiento etnográfico a la cultura escolar, Paidós, Madrid (2000).

Bourdieu, P. What Makes a Social Class? On the Theoretical and Practical Existence of Groups. Berkeley Journal of Sociology, 32: 1-17. (1987).

Burnett, C. y Merchant, G., Literacy-as-event: accounting for relationality in literacy research,

https://doi.org/10.1080/01596306.2018.1460318, Discourse: Studies in the Cultural Politics of Education, 1-12 (2018).

Burnett, C., Merchant, G., Pahl, K., y Rowsell, J. The (im) materiality of literacy: The significance of subjectivity to new literacies research, https://doi.org/10.1080/01596306.2012.739469, Discourse: Studies in the Cultural Politics of Education, 35(1), 90-103, (2014).

CIS. Centro de Investigaciones Sociológicas de España. Barómetro, Estudio nº 3149. (2016).

Coiro, J., Knobel, M., Lankshear, C., y Leu, D. J. (eds.). Handbook of research on new literacies, Routledge, (2014).

Csikszentmihalyi, M., y Nakamura, J. Flow, altered states of consciousness, and human evolution. Journal of Consciousness Studies, 25(11-12), 102-114 (2018).

Curwood, J. S., \& Cowell, L. L. H., iPoetry: Creating Space for New Literacies in the English Curriculum, https://doi.org/10.1002/JAAL.00014, Journal of Adolescent \& Adult Literacy, 55(2), 110-120 (2011).

Davies, J., (Im) material girls living in (im) material worlds: Identity curation through time and space. En C. Burnett, J. Davies, G. Merchant, \& J. Rowsell (Eds.), New literacies around the globe: Policy and pedagogy, pp. 72-87, New York, NY: Routledge (2014).

Feigenbaum, P., Collaborative imagination: Earning activism through literacy education, SIU Press (2015).

Fernández, M. y Martos, E., Cartografías lectoras y otros estudios de lectura. Lectura en las universidades públicas andaluzas, Editorial Marcial Pons (2014).

Forceville, C. y Urios-Aparisi, E. (Eds.). Multimodal metaphor. Walter de Gruyter (2009).

González, N., Moll, L. C., y Amanti, C. (Eds.), Funds of knowledge: Theorizing practices in households, communities, and classrooms, Routledge (2006).

Gutiérrez, A. C., Legarreta, O. E., y Garai, G. G., Integración de las TIC en la educación superior, Pixel-Bit. Revista de Medios y Educación, (21), 21-28 (2003).

Ibarra, N., y Ballester, J., La literatura infantil y juvenil en la formación del maestro, Lenguaje y textos, (38), 11-18 (2013).

Kell, C., Ariadne's thread: Literacy, scale and meaning-making across space and time. En Language, Literacy and Diversity (pp. 88-107), Routledge (2015).

Knobel, M., y Lankshear, C. (Eds.). A new literacies sampler, Peter Lang (2007).

Leander, K. M. y Ehret, C. Affect in Literacy Learning and Teaching: Pedagogies, Politics and Coming to Know. Routledge (2019).

Masgrau-Juanola, M., y Kunde, K., La intermedialidad: un enfoque básico para abordar fenómenos comunicativos complejos en las aulas, http://dx.doi.org/10.5209/ARIS.59812, Arte, Individuo y Sociedad, 30(3), 621-635 (2018).

Massumi, B., Politics of affect, John Wiley \& Sons (2015).

MECD Ministerio de Educación., Cultura y Deporte. Panorámica de la edición española de libros 2016, , Centro de Publicaciones del Ministerio (2016) 
Mills, K.A., Unsworth, L., DOI: 10.1093/acrefore/9780190264093.013.232, Multimodal Literacy. En G. Noblit (Ed.), Oxford Research Encyclopedia of Education, New York, Oxford University Press (2017).

Pahl, K., y Allan, C., 'I don't know what literacy is': Uncovering hidden literacies in a community library using ecological and participatory research methodologies with children, https://doi.org/10.1177/1468798411401864, Journal of Early Childhood Literacy, 11(2), 190-213 (2011).

Pahl, K., Materializing literacies in communities: The uses of literacy revisited, Bloomsbury Publishing (2014).

Pahl, K., y Rowsell, J., Artifactual literacies: Every object tells a story, Teachers College Press (2019).

Parry, B., Burnett, C., y Merchant, G. (Eds.), Literacy, media, technology: past, present and future, Bloomsbury Publishing (2016).

Ramírez, A., y Casillas Alvarado, M. A., Háblame de TIC: Tecnología digital en la educación superior, Córdoba, Editorial Brujas (2014).

Rockwell, E., La lectura como práctica cultural: conceptos para el estudio de los libros escolares, https://doi.org/10.1590/S1517-97022001000100002, Educação e pesquisa, 27(1), 11-26 (2001).

Rowsell, J., y Pahl, K. (eds.), The Routledge handbook of literacy studies, Routledge (2015).

Triviño, L., Principios Metodológicos de la Multimodalidad para la formación del profesorado de Ciencias Sociales. REIDICS: Revista de Investigación en Didáctica de las Ciencias Sociales, (3), 71-86 (2018).

Vergara, J. J., Aprendo porque quiero, Madrid, Ediciones SM (2017). 
